\title{
Ecosistemas para el emprendimiento: características del concepto y su aplicación a la empresa social
}

\author{
Jorge Antonio Torres Ortega \\ Universidad de Santiago de Chile/Universidad de Valencia. Doctorado en Economía Social \\ Académico Departamento de Tecnologías de Gestión, Universidad de Santiago de Chile \\ jorge.torres@usach.cl
}

DOI: $10.1387 /$ reves. 18004

Fecha de entrada: 23/04/2017

Fecha de aceptación: 15/06/2017

Sumario: 1. Introducción. 2. Elementos comunes al enfoque ecosistémico. 3. Distintos modelos de ecosistema para el emprendimiento. 4. Conclusiones. 5. Referencias bibliográficas.

\begin{abstract}
Resumen:
Las distintas aproximaciones que hacen uso del concepto biológico de ecosistema para abordar el fenómeno del emprendimiento subrayan la necesidad de capturar de manera holística todos los factores ambientales que contribuyen al éxito o fracaso de los emprendedores. Si bien este enfoque ha venido ganando una aceptación cada vez mayor entre académicos y policy makers, no puede afirmarse que exista un consenso en cuanto al significado exacto del constructo ecosistema para el emprendimiento. El presente artículo tiene como objetivo principal evaluar las similitudes y diferencias presentes en las distintas versiones del enfoque ecosistémico, con énfasis en el emprendimiento social. Se advierte que, aunque existen premisas comunes, los distintos modelos de ecosistema para el emprendimiento se diferencian en cuanto a los factores que destacan como condicionantes del surgimiento y permanencia de las iniciativas emprendedoras. En el caso del emprendimiento social, dichas diferencias tienen importantes implicaciones de carácter práctico.
\end{abstract}

\section{Palabras clave:}

Ecosistema, emprendimiento social, empresa social, enfoque sistémico. 


\begin{abstract}
:
The different approaches that make use of the biological concept of ecosystem to assess the phenomenon of entrepreneurship emphasize the need to capture in a holistic way all the environmental factors that contribute to the success or failure of entrepreneurs. While this approach has gained increasing acceptance among academics and policy makers, there is no consensus on the precise meaning of the ecosystem for entrepreneurship construct. This article aims to evaluate the similarities and differences present in the different versions of the ecosystem approach, with emphasis on social entrepreneurship. It is noticed that, although there are common premises, the different ecosystem models for entrepreneurship differ in terms of the factors that stand out as conditioners of the emergence and permanence of the entrepreneurial initiatives. In the case of social entrepreneurship, these differences have important practical implications.
\end{abstract}

\title{
Keywords:
}

Keywords: ecosystem, social entrepreneurship, social enterprise, systemic approach.

\section{Claves Econlit:}

L310, L290, M130, Z000.

\section{Introducción}

El fenómeno del emprendimiento social ha venido adquiriendo cada vez mayor importancia en nuestras sociedades (Nicolás, 2014). Este creciente interés se explica en gran medida por las múltiples crisis que han aquejado a diversos países y territorios alrededor del mundo, lo que ha llevado a depositar grandes esperanzas en este tipo de iniciativas para la solución de los acuciantes problemas sociales que éstas han traído consigo (Vernis y Navarro, 2011; Enciso, Gómez y Mugarra, 2012).

Es así como, desde diversos sectores, la empresa social han sido considerada como una respuesta innovadora, dinámica, inclusiva y autosostenible a los principales desafíos sociales, económicos y medioambientales que enfrentan las sociedades actuales, muchos de los cuales han sido desatendidos - ya sea por incapacidad y/o desinterés - tanto por los gobiernos y las instituciones públicas como por el sector privado (Thompson, Alvy y Lees, 2000; Santos, 2012; Defourny, Hulgrad y Pestoff, 2014; Nicolás, 2014; Caballero, Fuchs y Prialé, 2014; Farber, Caballero, Prialé y Fuchs, 2015; Comisión Europea, 2016). Sumado al cumplimiento de su misión social, estas empresas también cumplen un importante rol económico en tanto generan empleo y crecimiento (Vernis y Navarro, 2011). 
Es justamente en virtud del relevante impacto social y económico que generan las iniciativas empresariales en el ámbito del emprendimiento social, especialmente en momentos de crisis, que apoyar a los emprendedores sociales - sean estos actuales o potenciales - se ha convertido en una necesidad (Vernis y Navarro, 2011; Defourny, Hulgrad y Pestoff, 2014; Nicolás, 2014;). Lo anterior se ha visto reflejado en la puesta en marcha de una serie de políticas públicas destinadas a favorecer las actividades emprendedoras (Comisión Europea, 2016; Gómez, 2012). Como apuntan Vernis y Navarro (2011), de la promoción del éxito y la expansión de las ideas de los emprendedores sociales dependen en gran medida el nivel de competitividad de los países y el mejoramiento de las condiciones productivas y de vida de sus ciudadanos.

A diferencia de las empresas comerciales o convencionales, las empresas sociales tiene como objetivo primario generar valor social para los demás, proponiendo soluciones innovadoras a distintas problemáticas sociales a través de la aplicación de modelos de negocio orientados al mercado de bienes y servicios (Kliksberg, 2012; Caballero, Fuchs y Prialé, 2014; Farber, Caballero, Prialé y Fuchs, 2015). Es de esta manera como, por las características que les son propias, estas organizaciones enfrentan una serie de desafíos específicos en su accionar en el mercado, entre ellos el de su sostenibilidad, ya que, si quieren mantenerse en el tiempo y así provocar cambios sociales significativos y duraderos - con todos los beneficios que ello conlleva para los territorios en los que actúan-, deben generar no sólo valor social, sino que también valor económico (Vernis y Navarro, 2011).

Es así como el florecimiento y supervivencia de las empresas sociales depende de una multiplicidad de elementos. En general, los factores que favorecerían las iniciativas emprendedoras — sean éstas de tipo comercial o social - han sido extensamente tratados en la literatura sobre emprendimiento (Nicolás, 2014). En este sentido, un primer enfoque claramente identificable es aquel que se centra en el análisis del perfil psicológico y de los antecedentes personales del emprendedor, relevando como factores condicionantes del emprendimiento sus motivaciones, rasgos, formación, experiencia profesional, contexto familiar, valores y experiencias vividas, entre otros (Veciana, 2005).

Si bien el estudio de las características personales de los emprendedores resulta clave para comprender el proceso de creación de empresas (Baron, 2004; García, Martínez y Fernández, 2010), lo cierto es que también existen una serie de variables externas o del entorno que ayudarían a explicar el surgimiento de iniciativas emprendedoras (Shane y Venkataraman, 2000). De este modo, diversos autores han desarrollado modelos que destacan la influencia que tendrían factores tales como la 
tecnología, las normas sociales, la legislación y las políticas públicas (Langowitz y Minniti, 2007). En esta línea, en el último tiempo han ido tomando fuerza un conjunto de aproximaciones que también subrayan la importancia de los elementos ambientales o del entorno, aunque con pretensiones de alcance mayor. Estas visiones recalcan la necesidad de un modelo de emprendimiento que capture de manera holística todos los factores contextuales que contribuyen al éxito o fracaso de los emprendedores (Isenberg, 2011; Suresh y Ramraj, 2012). Con esta intención, varios autores han tomado prestado un término ampliamente utilizado en el campo de la biología para poner de manifiesto las limitaciones de estudiar a las organizaciones empresariales como entes aislados: el concepto de ecosistema.

La metáfora del ecosistema sirve para destacar que, al igual que los organismos vivos, las organizaciones empresariales establecen relaciones complejas con sus respectivos entornos, por lo tanto su devenir se encuentra condicionado por estos así como por el impacto mutuo que dichas organizaciones ejercen entren entre sí y hacia sus ambientes (Bloom y Dees, 2008). De este modo, las diversas aproximaciones que aquí agrupamos bajo el rótulo de enfoque ecosistémico tienen en común el hecho de entender el emprendimiento como un fenómeno complejo y multifactorial que involucra el reconocimiento del entorno y sus diversos actores (Bloom y Dees, 2008; García y Valencia, 2009; Isenberg, 2011; Caballero, Fuchs y Prialé, 2014). Este enfoque tiene importancia tanto teórica como práctica, ya que ha sido tomado desde distintas organizaciones para la generación de políticas tendientes al desarrollo de condiciones ambientales propicias para el estímulo de iniciativas emprendedoras (Comisión Europea, 2016). Ejemplo de ello son, en el área del emprendimiento social, las medidas propuestas desde la Unión Europea en orden a generar ecosistemas que promuevan la creación y supervivencia de empresas sociales en sus países miembros - especialmente en aquellos donde el Tercer Sector o sector de la Economía Social ha sido históricamente débil (Borzaga, Fazzi y Galera, 2016; Lambru y Petrescu, 2016) —, de tal manera de apuntar hacia un crecimiento económico inclusivo, sostenible e integrador (Enciso, Gómez y Mugarra, 2012; Comisión Europea, 2011, 2016).

La idea de ecosistema para el emprendimiento ha mostrado ser de utilidad a la hora de relevar los factores críticos del entorno de los cuales depende el éxito o fracaso de las iniciativas emprendedoras, ya sean comerciales o sociales (Stam y Spigel, 2016). Ahora bien, aunque los autores que desarrollan marcos analíticos centrados en este concepto destacan una serie de elementos comunes para la comprensión del accionar de las empresas en el mercado, lo cierto es que existen algunas diferen- 
cias conceptuales en cuanto a qué se entiende concretamente por ecosistema y a cuáles son sus componentes, lo que asimismo tiene importantes derivaciones prácticas. En este sentido, no se puede hablar de una definición ampliamente aceptada de ecosistema para el emprendimiento (Stam y Spigel, 2016). Por otra parte, el ecosistema y sus componentes adquieren rasgos distintivos en el caso del emprendimiento social, ya que estos emprendedores se enfrentan a entramados ambientales con características en muchos aspectos diferentes si se comparan con los ecosistemas en que se mueven los emprendedores comerciales (Vernis y Navarro, 2011).

El presente artículo pretende poner de relieve los elementos o ideas comunes así como las diferencias existentes entre las distintas versiones del enfoque ecosistémico, con énfasis en las implicancias de este concepto para el emprendimiento social.

\section{Elementos comunes al enfoque ecosistémico}

El concepto de ecosistema para el emprendimiento ha atraído mucha atención en el último tiempo, especialmente en círculos relacionados con las políticas públicas (Mack y Qian, 2016; Stam y Spigel, 2016). El término fue originalmente acuñado en el campo de la administración de empresas por Moore (1993: 26), quien lo define como una comunidad económica sostenida sobre la base de organizaciones e individuos que interactúan entre sí, la cual produce bienes y servicios de valor para los clientes, quienes a su vez también son parte del ecosistema junto con los proveedores, productores, competidores y otros actores relevantes. El autor busca destacar el hecho de que la empresas no operan en un vacío sino que, por el contrario, la forma en cómo interactúan con sus proveedores, clientes y financistas es de naturaleza relacional.

El desarrollo del actual enfoque ecosistémico — que se diferencia de la concepción de Moore (1993) por tratarse de una aproximación inherentemente geográfica y no centrada en la organización de una sola industria o cadena de valor - puede explicarse en parte como una reacción contra las explicaciones individualistas y centradas en los atributos de personalidad de los emprendedores, en dirección de una perspectiva más compleja que atendiera al rol que juegan las fuerzas sociales, culturales y económicas en el proceso empresarial (Nijkamp, 2003; Steyaert y Katz, 2004; Dodd y Anderson, 2007). Más específicamente, quienes defienden una perspectiva basada en la idea de ecosistema han recalcado la necesidad de comprender el emprendimiento dentro del contexto - tomado en un sentido amplio- en el que se despliega (Zahra y Wright, 2011; 
Autio, Kenney, Mustar, Siegel y Wright, 2014; Zhara, Wright y Abdelgawad, 2014), en contraste con las visiones que ponen su énfasis en las características y comportamientos de los individuos y las organizaciones (Shane y Venkataraman, 2000). Por otra parte, como ya adelantamos, la literatura sobre emprendimiento carecería de una perspectiva holística que considere la interrelación de todos los aspectos que dan forma a este fenómeno (Suresh y Ramraj, 2012; Isenberg, 2011). Además, se critica el hecho de que tradicionalmente las oportunidades empresariales hayan sido visualizadas como exógenas, vale decir, no considerando la creación de dichas oportunidades como algo inherente al proceso emprendedor (Qian, Acs y Stough, 2013).

La configuración de un ecosistema particular depende del complejo de interacciones que se establecen entre tres partes: individuos, organizaciones e instituciones. Dentro de este marco la figura del emprendedor ocupa el rol central, pues es él quien construye y sostiene el ecosistema (Borissenko y Boshma, 2017). En la tabla siguiente se puede observar una serie de definiciones de ecosistema de emprendimiento - distinguidas según el ámbito para el cual fueron pensadas (emprendimiento social o comercial)—, las que, más allá de ciertas diferencias, reconocen la interacción entre los componentes antes mencionados.

Las diferentes partes del ecosistema —individuos, organizaciones, instituciones- interactúan entre sí en formas complejas y específicas, configurando ecosistemas con características únicas (Isenberg, 2011). Por ejemplo, las instituciones — que de acuerdo con Sine y David (2010) incluyen tres dimensiones: normativa (normas y valores), cognitiva-cultural (refiere a cómo deben hacerse las cosas) y regulatoria (leyes y directrices) - pueden servir de apoyo en algunas regiones en mayor medida que en otras, pudiendo configurar distintos ritmos y tipos de actividad empresarial, así como diferentes caminos de desarrollo (Gertler, 2010).

Hasta aquí es posible advertir algunas características comunes propias del enfoque del ecosistema, como su énfasis en la interrelación, interdependencia y complementariedad de sus partes. Esta aproximación al emprendimiento enfatiza que este fenómeno se despliega en comunidades de actores que se implican e interactúan entre sí de maneras variadas, dependen los unos de los otros y se necesitan mutuamente para el logro de sus objetivos, generando sinergias y círculos virtuosos (Freeman y Audia, 2006; Vernis y Navarro, 2011). Esta interactividad pone de manifiesto otro rasgo importante del enfoque ecosistémico: los ecosistemas son inherentemente dinámicos, pues van cambiando y evolucionando con el tiempo. Y no sólo son dinámicos, sino que también flexibles y moldeables, ya que pueden ser creados o estimulados deliberadamente para favorecer el emprendimiento (Isenberg, 2011). 
Tabla 1

Definiciones de ecosistema

\begin{tabular}{|c|c|c|}
\hline Autor(es) & Ámbito & Definición \\
\hline $\begin{array}{l}\text { Stam y Spiegel } \\
(2017: 1)\end{array}$ & $\begin{array}{l}\text { Emprend. } \\
\text { comercial }\end{array}$ & $\begin{array}{l}\text { Conjunto de actores y factores interdepen- } \\
\text { dientes, coordinados de tal manera que } \\
\text { permiten el emprendimiento productivo } \\
\text { dentro de un territorio específico. }\end{array}$ \\
\hline $\begin{array}{l}\text { Mason y Brown } \\
(2014: 5)\end{array}$ & $\begin{array}{l}\text { Emprend. } \\
\text { comercial }\end{array}$ & $\begin{array}{l}\text { Conjunto interconectado de emprende- } \\
\text { dores (actuales y potenciales), organizacio- } \\
\text { nes empresariales, instituciones (tales como } \\
\text { universidades, agencias del sector público } \\
\text { y organismos financieros) y procesos em- } \\
\text { prendedores, los cuales formal e informal- } \\
\text { mente se fusionan para conectar, mediar } \\
\text { y dirigir el desempeño dentro del entorno } \\
\text { emprendedor local. }\end{array}$ \\
\hline $\begin{array}{l}\text { Qian, Acs y Stough } \\
(2013: 561)\end{array}$ & $\begin{array}{l}\text { Emprend. } \\
\text { comercial }\end{array}$ & $\begin{array}{l}\text { Factores económicos, sociales e institucio- } \\
\text { nales (así como cualquier otra clase de fac- } \\
\text { tores importantes) que interactivamente } \\
\text { influyen en la creación, descubrimiento y } \\
\text { explotación de oportunidades empresaria- } \\
\text { les. }\end{array}$ \\
\hline $\begin{array}{l}\text { Núñez, Jara y Mazo } \\
(2016: 50)\end{array}$ & $\begin{array}{l}\text { Emprend. } \\
\text { comercial }\end{array}$ & $\begin{array}{l}\text { Conjunto de actores (personas, organiza- } \\
\text { ciones, instituciones) y factores (cultura, } \\
\text { economía, regulación, etc.) que en su inter- } \\
\text { conexión y dinámica aumentan las proba- } \\
\text { bilidades de éxito de los emprendimientos. }\end{array}$ \\
\hline $\begin{array}{l}\text { Vera, Prialé, Fuchs, Espi- } \\
\text { nosa, Seminario y Nina- } \\
\text { huanca (2016: 344-345) }\end{array}$ & $\begin{array}{l}\text { Emprend. } \\
\text { social }\end{array}$ & $\begin{array}{l}\text { Comprensión de las dinámicas en las que } \\
\text { diferentes actores e instituciones partici- } \\
\text { pan, crean redes y favorecen un entorno } \\
\text { particular para emprender. }\end{array}$ \\
\hline $\begin{array}{l}\text { Bloom y Dees } \\
(2008: 47)\end{array}$ & $\begin{array}{l}\text { Emprend. } \\
\text { social }\end{array}$ & $\begin{array}{l}\text { Incluye a todos los actores (los amigos, } \\
\text { enemigos, competidores e incluso los es- } \\
\text { pectadores inocentes) así como el entorno } \\
\text { más amplio (las leyes, las políticas, las nor- } \\
\text { mas sociales, las tendencias demográficas y } \\
\text { las instituciones culturales) dentro del cual } \\
\text { estos juegan. }\end{array}$ \\
\hline
\end{tabular}

Fuente: elaboración propia. 


\section{Distintos modelos de ecosistema para el emprendimiento}

Como vimos, el enfoque ecosistémico se caracteriza por compartir una serie de premisas desde las cuales aborda el fenómeno emprendedor. Ahora bien, dentro de este marco analítico se han planteado una serie de modelos de ecosistema para el emprendimiento, los cuales se diferencian entre sí —en mayor o menor medida - desde el punto de vista de los elementos que los componen.

En el área del emprendimiento comercial, uno de los modelos de ecosistema más influyentes en los últimos ańos ha sido el desarrollado por Isenberg (2011), quien habla explícitamente de una «estrategia de ecosistema emprendedor para el desarrollo económico». La necesidad de una estrategia tal nace de la constatación de que ya se sabe lo suficiente acerca de cómo se desarrolla el emprendimiento en el mundo como para deliberadamente crear las condiciones para su estímulo, esto en un período de tiempo relativamente corto y sin grandes costos. Su modelo de ecosistema está compuesto por seis dominios: (i) Políticas (liderazgo, gobierno); (ii) Financiamiento (capital financiero); (iii) Cultura (normas sociales, historias de éxito); (iv) Apoyos (infraestructura, profesiones de apoyo, ONGs); (v) Capital Humano (trabajo, instituciones educacionales); y (vi) Mercados (clientes tempranos, redes). Estos dominios a su vez incluyen cientos de elementos interactuando en formas altamente complejas, por lo que a juicio del autor la identificación de patrones causales tiene un valor limitado, prefiriendo así enfatizar la importancia del contexto, en el sentido de que cada ecosistema emerge bajo un conjunto irrepetible de condiciones y circunstancias. Para Isenberg (2011) no existe una fórmula única para la creación de este ecosistema, por lo que plantea que la única manera de resolver el problema es de manera holística y específica, vale decir, impactando al ecosistema entero y estimulando círculos virtuosos entre todos sus elementos.

Por su parte, Spigel (2015) desarrolla un modelo que agrupa a los elementos constitutivos del ecosistema en tres categorías: (i) Atributos culturales (cultura e historias sobre emprendimiento propicias); (ii) Atributos sociales (talentos en el empleo, capital de inversión, redes, mentores y modelos a seguir); y (iii) Atributos materiales (políticas y gobernanza, universidades, servicios de apoyo, infraestructura física, mercados abiertos). Estos atributos no están aislados los unos de los otros, sino que se crean y reproducen por medio de sus interrelaciones.

Posteriormente, Spiegel y Stam (2016) amplían este modelo, distinguiendo dentro del ecosistema entre condiciones marco y condiciones sistémicas. Las primeras incluyen a las instituciones formales, la cultura, la infraestructura física y la demanda. Por otro lado, las condiciones sistémi- 
cas - que a juicio de los autores constituyen el corazón del ecosistema, en el sentido de que la presencia de estos elementos y la interacción entre ellos es crucial para su éxito- corresponden a las redes, el liderazgo, el financiamiento, los talentos, el conocimiento y los servicios de apoyo/intermediarios.

En suma, los distintos modelos de ecosistema para el emprendimiento comercial destacan los elementos que resultarían determinantes para el estímulo y persistencia de iniciativas emprendedoras de este tipo. En la literatura reciente en esta área es posible encontrar sistematizaciones que destacan los factores de mayor importancia para el éxito de un ecosistema, según han sido identificados y conceptualizados por diversos modelos explicativos; se subraya así la relevancia de contar con empleados talentosos en todos los sectores y áreas de expertise, de disponer de capital financiero, de que hayan emprendedores con capacidad de liderazgo así como mentores y consultores respetados por la comunidad, de que existan políticas públicas adecuadas así como culturas particulares que refuercen la actividad emprendedora, etc. (Feld, 2012; Spiegel y Stam, 2016; Borissenko y Boshma, 2017). En el campo de estudio del emprendimiento social también ha habido algunos esfuerzos — aunque menos sistemáticos- por identificar las características que debería tener un ecosistema que permita el florecimiento y la sostenibilidad de las empresas sociales. Algunos de los componentes reconocidos como importantes para los ecosistemas de emprendimiento convencionales también lo son para los ecosistemas de emprendimiento social, aunque aquí cuentan con una serie de especificidades (Vernis y Navarro, 2011).

En este sentido, Vernis y Navarro (2011) afirman que, para que los emprendedores sociales puedan actuar en el mercado en igualdad de condiciones que el resto de las empresas, estos deben contar con un ecosistema de emprendimiento social que tenga al menos seis elementos, según se detalla en la Tabla 2.

De la propuesta de estos autores se desprende que los emprendedores sociales requieren de apoyos más específicos que los emprendedores comerciales, dadas las características particulares que reviste el emprendimiento social (mayores dificultades a la hora de conseguir financiamiento, menor conocimiento de parte de los emprendedores sociales respecto de cómo funcionan los mercados, etc.). Ahora bien, al igual que en el emprendimiento convencional, Vernis y Navarro (2011) señalan que es necesario apoyar todos estos elementos de manera holística, vale decir, el mantenimiento del ecosistema de emprendimiento social a largo plazo dependerá de hacer avanzar todos estos aspectos de manera conjunta y no aisladamente. 
Tabla 2

Ecosistema de emprendimiento social de Vernis y Navarro (2011)

\begin{tabular}{|c|c|}
\hline Componentes & Definiciones \\
\hline $\begin{array}{l}\text { Formación e } \\
\text { investigación }\end{array}$ & $\begin{array}{l}\text { Necesidad de que existan centros de formación que acompañen a } \\
\text { los emprendedores sociales en su actuar en el mercado, y que con- } \\
\text { tribuyan a crear una "cultura» del sector que permita el fortaleci- } \\
\text { miento institucional de estas organizaciones. }\end{array}$ \\
\hline Asesoramiento & $\begin{array}{l}\text { Los emprendedores sociales requieren de otras organizaciones que } \\
\text { les acompañen durante sus fases de desarrollo en el mercado (idea- } \\
\text { inicio-desarrollo-escala), ya que son muchos los aspectos a los estos } \\
\text { deben prestar atención cuando inician una empresa social. }\end{array}$ \\
\hline Financiación & $\begin{array}{l}\text { Dado que conseguir capital para poner en marcha una empresa no } \\
\text { es algo fácil - especialmente en el caso de un emprendimiento so- } \\
\text { cial-, la financiación es una pieza fundamental en cualquier eco- } \\
\text { sistema de emprendimiento social si se quiere que los emprende- } \\
\text { dores avancen en el mercado. }\end{array}$ \\
\hline Innovación & $\begin{array}{l}\text { Resulta muy importante lograr una innovación constante en el } \\
\text { emprendimiento social, por ello es importante que existan centros } \\
\text { dedicados a hacer avanzar esta parte del ecosistema. }\end{array}$ \\
\hline Redes & $\begin{array}{l}\text { La creación de redes de emprendedores sociales — tanto formales } \\
\text { como informales- es muy importante para el mantenimiento del } \\
\text { ecosistema a largo plazo. El apoyo a un emprendedor social puede } \\
\text { provenir de diversos campos, e incluso de los propios emprende- } \\
\text { dores sociales. }\end{array}$ \\
\hline Difusión & $\begin{array}{l}\text { Con el objetivo de crear una cultura del emprendimiento social } \\
\text { que ayude a mantener y acrecentar el espíritu emprendedor, así } \\
\text { como para hacer aflorar en la sociedad esta nueva punta del cono- } \\
\text { cimiento y tener mayor presencia y apoyo en ella, resulta funda- } \\
\text { mental comunicar y difundir todo lo que sucede en torno al em- } \\
\text { prendimiento social. }\end{array}$ \\
\hline
\end{tabular}

Fuente: elaboración propia en base a Vernis y Navarro (2011).

Los elementos identificados en el modelo elaborado por los autores citados coinciden en parte con el plan de acción propuesto por la Comisión Europea en 2011 para estimular el desarrollo de las empresas sociales, el que cual incluye tres grandes objetivos, a saber: mejorar la visibilidad de este sector, su entorno jurídico y su acceso a la financiación (Comisión Europea, 2011; Enciso, Gómez y Mugarra, 2012). Esta propuesta encuen- 
tra un mayor desarrollo en un documento reciente (Comisión Europea, 2016), en el cual se reconoce el carácter complejo de los ecosistemas para el emprendimiento social. Dichos ecosistemas se sostendrían en dos pilares fundamentales: (i) en políticas públicas que reconozcan, regulen y apoyen a las empresas sociales, en vista de su multiplicación; y (ii) en la habilidad de los ciudadanos para auto-organizarse, lo que conduciría a la emergencia y desarrollo de empresas sociales desde «abajo hacia arriba». A su vez, estos dos pilares dan forma e influyen en un conjunto de factores evolutivos e interrelacionados que configuran lo que la Comisión Europea (2016) entiende que debe incluir un adecuado ecosistema para el emprendimiento social (Tabla 3).

Tabla 3

Ecosistema de emprendimiento social de la Comisión Europea (2016)

\begin{tabular}{|c|c|}
\hline Componentes & Sub-componentes \\
\hline $\begin{array}{l}\text { Reconocimiento político } \\
\text { y formas legales }\end{array}$ & $\begin{array}{l}\text { Legislaciones específicas para las empre- } \\
\text { sas sociales; creación de unidades mi- } \\
\text { nisteriales, estructuras y departamentos } \\
\text { a cargo de promover las empresas socia- } \\
\text { les; referencias explícitas a las empresas } \\
\text { sociales en documentos oficiales; adop- } \\
\text { ción de estrategias nacionales o regiona- } \\
\text { les/locales específicas sobre empresas so- } \\
\text { ciales, etc. }\end{array}$ \\
\hline Acceso al mercado & $\begin{array}{l}\text { Tipo de relaciones que las empresas esta- } \\
\text { blecen con los sistemas públicos de bien- } \\
\text { estar; políticas públicas de promoción, } \\
\text { integrativas y/o sustitutivas, etc. }\end{array}$ \\
\hline $\begin{array}{l}\text { Apoyo público para la puesta en marcha } \\
\text { y ampliación de las empresas sociales }\end{array}$ & $\begin{array}{l}\text { Incentivos fiscales específicos para las } \\
\text { empresas sociales, etc. }\end{array}$ \\
\hline Acceso a financiamiento & $\begin{array}{l}\text { Instrumentos de financiamiento innova- } \\
\text { dores; programas que favorezcan la capi- } \\
\text { talización, etc. }\end{array}$ \\
\hline Redes y mecanismos de apoyo mutuo & $\begin{array}{l}\text { Movimientos de empresas sociales; redes } \\
\text { de emprendedores sociales, etc. }\end{array}$ \\
\hline $\begin{array}{l}\text { Desarrollo de la investigación, } \\
\text { la educación y las habilidades }\end{array}$ & $\begin{array}{l}\text { Capacitación en emprendimiento social; } \\
\text { estudios sobre empresas sociales, etc. }\end{array}$ \\
\hline
\end{tabular}

Fuente: elaboración propia en base a Comisión Europea (2016). 
En contraste con los modelos de Vernis y Navarro (2011) y de la Comisión Europea (2016), los cuales - a pesar de reconocer la importancia del tejido social y de la auto-organización de los ciudadanos- parecen articularse mejor con estrategias de promoción del emprendimiento social conducidas desde «arriba hacia abajo», Bloom y Dees (2008) presentan una propuesta de ecosistema cuyo centro es el sujeto emprendedor. En este sentido, el modelo de estos autores responde a la necesidad de los emprendedores sociales de alterar el ecosistema en su beneficio, de modo que puedan alcanzar sus objetivos y así generar cambios sociales significativos y duraderos. El modelo en cuestión se traduce de esta manera en una herramienta con la cual los emprendedores sociales pueden crear un «mapa» de su ecosistema, en el que deben identificar todos los actores (individuos y organizaciones) y las condiciones ambientales (normas, mercados, leyes) que lo configuran, así como las relaciones entre estos, que podrían llegar a afectar su posibilidad de crear y sostener el impacto pretendido por su organización. El detalle de esta conceptualización puede observarse en la Tabla 4.

\section{Tabla 4}

Ecosistema de emprendimiento social de Bloom y Dees (2008)

\begin{tabular}{c|l}
\hline \multicolumn{1}{c|}{ Componentes } & \multicolumn{1}{c}{ Sub-componentes } \\
\hline \multirow{3}{*}{ Actores } & - Proveedores de recursos. \\
& - Competidores. \\
& - Aliados y organizaciones complementarias. \\
& - Beneficiarios y clientes. \\
& - Oponentes y actores problemáticos. \\
& - Espectadores influyentes o afectados. \\
\hline Condiciones ambientales & - Políticas y estructuras administrativas. \\
& - Economía y mercados. \\
& - Cultura y tejido social. \\
\hline
\end{tabular}

Fuente: elaboración propia en base a de Bloom y Dees (2008).

En línea con el enfoque ecosistémico, los modelos revisados reconocen el carácter holístico, interdependiente, interrelacionado, dinámico y permeable de los ecosistemas de emprendimiento social. Ahora bien, la propuesta de Bloom y Dees (2008) encaja mejor con estrategias individuales de alteración del ecosistema, mientras que en el caso de Vernis y Navarro (2011) y la Comisión Europea (2016) sus modelos responden primariamente a la necesidad de generar las condiciones institucionales adecuadas 
para el surgimiento y promoción de las empresas sociales. Por otra parte, existen investigaciones realizadas desde el campo del emprendimiento social que, aunque se apropian del concepto de ecosistema con las características que hemos visto hasta ahora, lo utilizan fundamentalmente con la finalidad de realizar un diagnóstico de la posibilidad de este tipo de emprendimiento en contextos específicos. Ejemplo de esto son los trabajos de Vera, Prialé, Fuchs, Espinosa, Seminario y Ninahuanca (2016) y de Heri y Sutanti (2016), los cuales buscan entender y evaluar las condiciones sistémicas que permiten o limitan las iniciativas emprendedoras de carácter social en Lima (Perú) e Indonesia, respectivamente.

\section{Conclusiones}

Existen una serie de elementos comunes que subyacen a las aproximaciones que se apropian del concepto de ecosistema para abordar el análisis del emprendimiento en el ámbito tanto comercial como social. Los ecosistemas constituyen entramados interactivos, dinámicos y permeables en los cuales individuos, organizaciones e instituciones establecen complejas relaciones de interdependencia y complementariedad. Ahora bien, más de allá de este sustrato común, los diferentes modelos de ecosistema para el emprendimiento presentan diferencias en cuanto a los factores identificados como condicionantes de la emergencia y sostenibilidad de las iniciativas emprendedoras. Comparativamente, los modelos de ecosistema para el emprendimiento comercial y social coinciden en la identificación de algunos de estos aspectos (necesidad de financiamiento, políticas adecuadas, etc.), aunque por las características propias de este primer tipo de emprendimiento dichos factores revisten ciertos rasgos distintivos. Por otra parte, los modelos de ecosistema para el emprendimiento social analizados, aunque parten de las mismas premisas, se distinguen por cuanto refieren a distintas estrategias y usos prácticos, como son la necesidad de generar condiciones institucionales propicias para la empresa social, de diseñar planes de acción individuales para alterar el propio ecosistema, y de generar diagnósticos ad hoc en contextos específicos.

Como señalan Borissenko y Boshma (2017), a pesar del creciente interés que ha generado el enfoque ecosistémico, éste presenta una serie de limitaciones, como es la falta de un marco analítico que haga explícitas las conexiones causales existentes entre los distintos componentes de un ecosistema. No obstante ello, para el caso del emprendimiento social este enfoque ha mostrado ser de utilidad por cuanto pretende captar el surgimiento y persistencia de este fenómeno en su toda su complejidad, a la vez que se presta particularmente bien para la elaboración de estrategias tendientes al fortalecimiento y consolidación de las empresas sociales. 


\section{Referencias bibliográficas}

AUTIO, E., KENNEY, M., MUSTAR, P., SIEGEL, D. y WRIGHT, M. (2014): «Entrepreneurial innovation: The importance of context», Research Policy, vol. 43, n. ${ }^{\circ}$ 7, págs. 1097-1108.

BARON, R. (2004): «The cognitive perspective: A valuable tool of answering entrepreneurship's basic 'why' questions», Journal of Business Venturing, n. ${ }^{\circ} 19$, págs. 221-240.

BLOOM, P. y DEES, G. (2008): «Cultivate your ecosystem», Stanford Social Innovation Review, Winter, págs. 47-53.

BORISSENKO, J. y BOSHMA, R. (2017): «A critical review of entrepreneurial ecosystems research: towards a future research agenda", Paper in Innovation Studies, n. ${ }^{\circ}$ 3, Centre for Innovation, Research and Competence in the Learning Economy (CIRCLE), Lund University, Sweden.

BORZAGA, C., FAZZI, L. y GALERA, G. (2016): «Social enterprise as a bottom-up dynamic. Part 1 . The reaction of civil society to unmet social needs in Italy, Sweden and Japan", International Review of Sociology, vol. 26, n. ${ }^{\circ} 1$, págs. $1-18$.

CABALLERO, S., FUCHS, R.M. y PRIALÉ, M.A. (2014): «The influence of the Big 5 personality traits on the social enterprise start-up intentions: A Peruvian case», Taylor's Business Review, vol. 4, n. ${ }^{\circ} 1$, págs. 1-18

COMISIÓN EUROPEA (2011): «Comunicación de la Comisión, Iniciativa en favor del emprendimiento social: Construir un ecosistema para promover las empresas sociales en el centro de la economía y la innovación sociales», n. ${ }^{\circ} 682$.

COMISIÓN EUROPEA (2016): Social enterprises and their eco-systems: developments in Europe, Publications Office of the European Union, Luxembourg.

DODD, S.D. y ANDERSON, A.R. (2007): «Mumpsimus and the mything of the individualistic entrepreneur», International Small Business Journal, n. ${ }^{\circ} 25$, págs. 341-360.

ENCISO, M., GÓMEZ, L. y MUGARRA, A. (2012): «La iniciativa comunitaria en favor del emprendimiento social y su vinculación con la economía social: una aproximación a su delimitación conceptual», CIRIEC-España, Revista de Economía Pública, Social y Cooperativa, n.o 75, págs. 54-80.

FARBER, V., CABALLERO, S., PRIALÉ, M. y FUCHS, R.M. (2015): «Social enterprises in Lima: Notions and operating models, Journal of Entrepreneurship and Innovation in Emerging Economies», vol. 1, n. ${ }^{\circ}$ 1, págs. 56-78.

FELD, B. (2012): Startup communities: Building an entrepreneurial ecosystem in your city, Wiley, New York.

FREEMAN, J.H. y AUDIA, P.G. (2006): «Community ecology and the sociology of organizations», Annual Review of Sociology, págs. 145-169.

GARCÍA, C., MARTÍNEZ, A. y FERNÁNDEZ, R. (2010): «Características del emprendedor influyentes en el proceso de creación empresarial y en el éxito esperado», Revista Europea de Dirección y Economía de la Empresa, vol. 19, n. ${ }^{\circ}$, págs. 31-48. 
GERTLER, M.S. (2010): "Rules of the game: the place of institutions in regional economic change», Regional Studies, vol. 44, n. ${ }^{\circ} 1$, págs. 1-15.

GÓMEZ, J.M. (coord.) (2012): Manual de casos sobre creación de empresas en España, McGrawHill, España.

HERI, A. y SUTANTI, A. (2016): «The ecosystem of social entreprise: Social culture, legal framework, and policy review in Indonesia», Pacific Science Review B: Humanities and Social Sciences, n. ${ }^{\circ}$ 2, págs. 106-112.

HERRERO, M. (2015): Las empresas sociales: un grupo en expansión en el ámbito de la Economía Social. Identificación y análisis de las características identitarias de la empresa social europea y su aplicación a la realidad de los Centros Especiales de Empleo de la economía española, Tesis de Doctorado, Programa Oficial de Doctorado en Economía Social (Cooperativas y Entidades No Lucrativas), Instituto Universitario de Economía Social y Cooperativa, Universidad de Valencia, España.

ISENBERG, D. (2011): The entrepreneurship strategy as a new paradigm for economic policy: Principles for cultivating entrepreneurship, presentación en el Institute of International and European Affairs, 12 de mayo, Dublín, Irlanda.

KLIKSBERG, B. (2012): Emprendedores sociales, los que hacen la diferencia, Grupo Editorial, Buenos Aires.

LAMBRU, M. y PETRESCU, C. (2016): «Bottom-up social enterprises in Romania. Case study - Retirees' Mutual Aid Associations», International Review of Sociology, vol. 26, n. ${ }^{\circ}$ 2, págs. 247-61.

LANGOWITZ, N. y MINNITI, M. (2007): "The entrepreneurial propensity of women», Entrepreneurship Theory and Practice, vol. 31, n.o 3, págs. 341-364.

LÓPEZ DE TORO, C. (2014): Características de emprendimiento social de los jóvenes en estudios previos a los universitarios, Trabajo de fin de Máster, Área de Recursos Humanos, Departamento de Organización de Empresas, Facultad de Ciencias Económicas y Empresariales, Universidad Complutense de Madrid, España.

MASON, C. y BROWN, R. (2014): Entrepreneurial ecosystems and growth oriented entrepreneurship, trabajo preparado para el taller organizado por el Programa OECD LEED y el Ministerio Holandés de Asuntos Económicos, La Haya, Holanda.

MOORE, J. (1993): «Predators and prey: A new ecology of competition», Harvard Business Review, May/June, págs. 75-86.

NICOLÁS, C. (2014): Emprendimiento social, Tesis de Doctorado, Departamento de Organización de Empresas y Finanzas, Universidad de Murcia, España.

NIJKAMP, P. (2003): «Entrepreneurship in a modern network economy», Regional Studies, n. ${ }^{\circ}$ 37, págs. 395-405.

NÚNEZZ, I., JARA, F. y MAZO, V. (2016): Ecosistemas de emprendimiento. Una mirada desde la política pública, CORFO, Gobierno de Chile.

MACK, E.A.y QIAN, H. (eds.) (2016): Geographies of entrepreneurship, Routledge, New York. 
QIAN, H., ACS, Z.J. y STOUGH, R.R. (2013): «Regional systems of entrepreneurship: the nexus of human capital, knowledge and new firm formation", Journal of Economic Geography, vol. 13, n.o 4, págs. 559-587.

ROY, M.J., McHUGH, N., HUCKFIELD, L., KAY, A. y DONALDSON, C. (2015): "The most supportive environment in the world"? Tracing the development of an institutional 'ecosystem' for social enterprise», Voluntas, n. ${ }^{\circ}$ 26, págs. 777-800.

SANTOS, F. (2012): Let's get serious about social entrepreneurship: re-thinking strategy and organization theory, 9th Annual NYU Social Entrepreneurship Research Conference, November.

SPIGEL, B. (2015): "The relational organization of entrepreneurial ecosystems», Entrepreneurship Theory and Practice, vol. 41, n. ${ }^{\circ}$ 1, págs. 49-72.

STAM, E. y SPIGEL, B. (2016): «Entrepreneurial ecosystems», Discussion Paper Series, 16-13, Tjalling C. Koopmans Research Institute, Utrecht School of Economics, Utrecht University.

STEYAERT, C. y KATZ, J. (2004): «Reclaiming the space of entrepreneurship in society: Geographical, discursive and social dimensions», Entrepreneurship and Regional Development, n. ${ }^{\circ}$ 16, págs. 179-196.

SHANE, S. y VENKATARAMAN, S. (2000): «The promise of entrepreneurship as a field of research", Academy of Management Review, n. ${ }^{\circ}$ 25, págs. 217226.

SINE, W.D. y DAVID R.J. (2010): "Institutions and entrepreneurship», Research in the Sociology of Work, n. ${ }^{\circ}$ 21, págs. 1-26.

SURESH, J. y RAMRAJ, R. (2012): «Entrepreneurial ecosystem: Case study on the influence of environmental factors on entrepreneurial success", European Journal of Business and Management», vol. 4, n. ${ }^{\circ}$ 16, págs. 95-101.

THOMPSON, J.L., ALVY, G. y LEES, A. (2000): «Social entrepreneurship: A new look at the people and the potential», Management Decision, vol. 38, n. ${ }^{\circ}$, págs. $328-338$.

VECIANA, J.M. (2005): La creación de empresas. Un enfoque gerencial, Servicio de Estudios, Caja de Ahorros y Pensiones de Barcelona, Barcelona, España.

VERA, A., PRIALÉ, M.A., FUCHS, R.M., ESPINOSA, A., SEMINARIO, M. y NINAHUANCA, E. (2016): «Hacia una comprensión del ecosistema emprendedor social peruano: contexto y características del emprendimiento social en Lima», Ciências Sociais Unisinos, vol. 52, n.o 3, págs. 343-353.

VERNIS, A. y NAVARRO, C. (2011): «El concepto de ecosistema para el emprendimiento social», Revista Espańola del Tercer Sector, n. ${ }^{\circ}$ 17, págs. 67-84.

ZAHRA, S.A., WRIGHT, M. y ABDELGAWAD, S.G. (2014): «Contextualization and the advancement of entrepreneurship research», International Small Business Journal, vol. 32, n. ${ }^{\circ}$ 5, págs. 479-500. 